\title{
Differences in Bleeding Episodes in Severe Hemophilia A Based on Nutritional Status
}

\author{
Wenny Simamora, ${ }^{1}$ Susi Susanah, ${ }^{2}$ Amaylia Oehadian ${ }^{3}$ \\ ${ }^{1}$ Faculty of Medicine Universitas Padjadjaran, Indonesia, ${ }^{2}$ Department of Child Health Faculty \\ of Medicine Universitas Padjadjaran/Dr. Hasan Sadikin General Hospital Bandung, Indonesia, \\ ${ }^{3}$ Department of Internal Medicine Faculty of Medicine Universitas Padjadjaran/Dr. Hasan Sadikin \\ General Hospital Bandung, Indonesia
}

\section{Abstract}

Background: Hemophilia A is a congenital bleeding disorder caused by the deficiency of coagulation factor VIII and is characterized by joint bleeding, especially in weight-bearing joints. An excess weight may cause bleeding in hemophilia due to increased joint tension. This study aimed to determine the differences in bleeding episodes between severe hemophilia A patients with and without excess weight.

Methods: A comparative observational analytic study was conducted in March-November 2019 using a cross-sectional design. Subjects were patients with severe hemophilia A registered in the Indonesian Hemophilia Society Association in West Java and had had severe hemophilia A for at least two years old with complete data on date of birth, height, weight, and bleeding intensity. Subjects were divided into groups with excess weight (excess weight) and without excess weight (non-excess weight). Nutritional status in adults was determined based on age-specific percentile BMI. Bleeding episodes were determined as frequency of bleeding in one year. The Mann-Whitney statistical test was used to observe the difference between groups.

Results: Of 226 severe hemophilia A patients registered, only 155 patients were included. Of these, 121 patients did not have excess weight and 34 had excess weight. The median bleeding episodes of in the non-excess weight and excess weight groups were 24(1-48) and 24(8-48), respectively ( $p=0.761)$. Conclusions: There is no difference in bleeding episodes between severe hemophilia A patients with excess weight and without excess weight. However, good nutrition education needs to be provided to these patients since excess weight may increase bleeding in joints.

Keywords: Bleeding episodes, excess weight, severe hemophilia A

\section{Introduction}

Hemophilia is a congenital bleeding disorder caused by a deficiency of coagulation factors. The deficiency of certain coagulation factors determines the hemophilia type. Deficiency of coagulation factor VIII is known as hemophilia $A$ and deficiency of coagulation factor IX as hemophilia B. ${ }^{1}$ Survey conducted by the World Federation of Hemophilia in 2017 has reported that there are 315,423 individuals with bleeding disorders throughout the world, including 196,706 people with hemophilia A. ${ }^{2}$ Hemophilia in Indonesia accounts for
2,054 patients. $^{2}$ Hemophilia is characterized by spontaneous joint bleeding and excessive bleeding triggered by among others an injury. ${ }^{3}$ Hemophilia is classified based on severity into mild, moderate, and severe hemophilia. ${ }^{1}$

People with hemophilia (PWH) especially those with severe hemophilia can experience spontaneous muscle and joint bleeding, ${ }^{1}$ usually in the lower limbs, which are weightbearing joints. ${ }^{4}$ The risk of being overweight in people with hemophilia ( $\mathrm{PWH}$ ) increases as a result of physical inactivity due to over protection from the parents. ${ }^{5}$ Excessive body weight increases the risk of joint bleeding

Correspondence: Wenny Simamora, Faculty of Medicine, Universitas Padjajaran, Jalan Raya Bandung Sumedang KM 21, Jatinangor, Sumedang Indonesia, E-mail: wennyahs@gmail.com 
in PWH who often experience arthropathy and can cause bleeding in hemophilia due to increased joint tension. ${ }^{5}$ The higher the body weight, the greater the mechanical stress that is imposed on the joints, especially weightbearing joints, causing damage to the tissues and connective structures of the joints. ${ }^{6}$

Research conducted in the Netherlands showed that there was a decrease in the hemophilia activities list (HAL) score, increased bleeding, and the use of factor VIII with a higher concentration in hemophilia patients who were overweight. ${ }^{7}$ Using the HAL score, hemophilia patients with overweight have an impaired functional ability compared with hemophilia patients with normal weight. ${ }^{7}$ A higher frequency of bleeds and a trend towards higher use of FVIII concentrate is also observed. ${ }^{7}$ There has been no research in Indonesia about differences in bleeding episodes based on nutritional status in people with hemophilia. Therefore, our study aimed to determine differences in bleeding episodes between severe hemophilia A patients with and without excess weight.

\section{Methods}

A comparative observational analytic study was conducted in March-November 2019 using a cross-sectional design. Subjects were those with severe hemophilia A registered in the Indonesian Hemophilia Society Association in West Java. Inclusion criteria were severe hemophilia A with complete data, i.e. date of birth, height and weight, and bleeding intensity, and at least two years old since the use of BMI charts was not recommended for children under two years old according to the CDC. Exclusion criteria were incomplete data, had died during the study, or otherwise could not be contacted. The minimum sample size needed per group was 25 subjects, with the choice of alpha significance level $(\alpha)=5 \%$, beta $(\beta)=5 \%$, the combined standard deviation $(S)=$ 2.948 and the mean difference (X1-X2) $=3 \mathrm{~kg} /$ $\mathrm{m}^{2}$. This study was approved by the Research Ethics Committee Universitas Padjajaran, with ethical number 1270/UN6.KEP/EC/2019.

The variables assessed were nutritional status as independent variables and bleeding episodes as dependent variables. Bleeding episodes were designated as the frequency of manifestations of bleeding experienced by the subject in one year. Several characteristics of the subjects, consisting of age, age of diagnosis, manifestations of bleeding, and nutritional status were described. The age was the group was classified into a group of children (2-18 years) and adults ( $\geq 18$ years) based on different ways of determining nutritional status. Nutritional status in adults was determined based on body mass index (BMI), which was the ratio of body weight in kilograms to the square of height in meters. In children, nutritional status was based on the BMI percentile, which was specific to age and sex. ${ }^{9}$ The subjects were classified into two groups, those with excess weight and the other with no excess weight. The excess weight group was subjects with overweight and obesity whereas the non-excess weight group was subjects with underweight and normal weight. The height and weight were measured at the last hospital visit. The data were then plotted to determine the nutritional status.

The episodes of bleeding were measured from the time the subject was contacted up to the one-year back. Measurement of bleeding intensity was counted on how many manifestations of bleeding had appeared in the past year. The collected data were processed using Microsoft Excel and IBM SPSS v.25.0. The data normality was tested using the Shapiro-Wilk test to determine the distribution of various variables. The MannWhitney statistical test was used to determine the difference in bleeding episodes in the nutritional status group. The significance of the hypothesis test was based on $p<0.05$. Data analysis results were presented in tables and figures.

\section{Results}

Of 338 people with hemophilia in West Java, 226 were severe A hemophilia and only 155 subjects met the inclusion criteria. Most people with hemophilia A were under 18 years old $(67.1 \%)$ of whom $63.9 \%$ was diagnosed after one-year-old. The most frequent manifestation of bleeding experienced by PWH was hemarthrosis (99.4\%), while the least was intracranial hemorrhage (14.2\%) (Table 1). The median of episodes of subject bleeding was 24 (range 1-8).

The bleeding episodes were shown based on the nutritional status of people with severe hemophilia A who had excess weight and non-excess weight (Table 2). The result showed a p-value of 0.761 , suggesting there was no significant difference in the bleeding episodes of people with severe hemophilia A who had excess weight and non-excess weight. However, those with excess weight had tended to have more bleeding episode with a minimum 
Table 1 Characteristics of People with Hemophilia Registered in the Indonesian Hemophilia Society Association in West Java

\begin{tabular}{lc}
\hline \multicolumn{1}{c}{ Characteristics } & $\mathbf{N}(\mathbf{\%})$ \\
\hline Age group & $104(67.1)$ \\
2-18 years old & $51(32.9)$ \\
$>18$ years old & \\
Diagnosis age group & $51(32.9)$ \\
$<1$ year old & $99(63.9)$ \\
$\geq 1$ year old & $5(3.2)$ \\
Unknown & $109(70.3)$ \\
Bleeding manifestation & $99(63.9)$ \\
Ecchymosis & $154(99.4)$ \\
Hematoma & $75(48.4)$ \\
Hemarthrosis & $139(89.7)$ \\
Epistaxis & $22(14.2)$ \\
Gum bleeding & $56(36.1)$ \\
Intracranial bleeding & $97(62.6)$ \\
Multiple hematomas & $110(71.0)$ \\
Hemarthrosis-hematoma & \\
Hemarthrosis-ecchymosis & $121(78.1)$ \\
Nutritional status & $34(21.9)$ \\
Non-excess weight & \\
Excess weight &
\end{tabular}

bleeding episode of 8 and a max of 48 .

\section{Discussion}

Our study has explored people with hemophilia $(\mathrm{PWH})$ registered in the Indonesian Hemophilia Society Association in West Java in relation to the period of bleeding. The median age of the PWH was 14 years old (range 2-43), and the majority was pediatric patients (2-18 years old). In another study, adult patients are more prevalent (70\%). ${ }^{10}$ This difference might be due to the availability of clotting factors and the costs required that are still obstacles in the prophylaxis protocol in Indonesia and other developing countries. ${ }^{11}$ In more developed countries, there is a higher percentage of adult patients since the prophylaxis is available, which is one of the factors that increase the life expectancy of people with hemophilia. ${ }^{2,9}$ Our study shows that the age of diagnosis of people with severe hemophilia $A$ is above one year old (63.9\%). This result is opposite to other studies showing that the manifestations in people with severe hemophilia would appear before one year old, especially when the period of learning to crawl and walk.12 This difference can be due to the lack of public knowledge about hemophilia. Some consider the cause of bleeding symptoms is due to

Table 2 Episodes of Bleeding among People with Severe Hemophilia A Based on Nutritional Status

\begin{tabular}{lcc}
\hline \multicolumn{1}{c}{ Nutritional status } & $\begin{array}{c}\text { Median bleeding episode } \\
\text { (min-max) }\end{array}$ & P-value \\
\hline Non-excess weight & $24(1-48)$ & $0.761^{*}$ \\
Excess weight & $24(8-48)$ & \\
Total & & \\
\hline
\end{tabular}

Note: * Mann-Whitney test 
another disorder/disease or because of a lack of health facilities to diagnose hemophilia. ${ }^{13}$

The most bleeding manifestation experienced by the subject is hemarthrosis (99.4\%), which is consistent with another study. ${ }^{14}$ Hemarthrosisismostoften experienced because this type of bleeding can also occur spontaneously when PWH performs routine activities as usual. ${ }^{14}$ Bleeding occurs because small capillaries in the synovial membrane rupture, causing joints to become stressed, and the synovial membrane is squeezed and pulled, ${ }^{14}$ as well as when a person performs routine activities, such as walking, climbing stairs, or bumping into something. ${ }^{14}$ Someone with a normal coagulation system can prevent bleeding, but not in PWH. ${ }^{14}$ The least bleeding manifestation experienced in $\mathrm{PWH}$ is intranial hemorrhages (14.2\%). Intracranial hemorrhage usually occurs due to spontaneous trauma, although in a small proportion..$^{12}$ The intracranial hemorrhage is also a significant cause of death in $\mathrm{PWH}^{1,12}$

There is thus no significant difference $(p=0.791)$ in bleeding episodes based on nutritional status of people with severe hemophilia $\mathrm{A}$, however, there is a trend that PWH with excess weight had more bleeding episodes. This is similar to the study showing that no difference in bleeding episodes in people with hemophilia who had excess weight and those who had not. ${ }^{8}$ However, association has been shown in the Netherlands $(p=0.045) . .^{7}$ The difference could be due to the small number of subjects, or any other factors related to bleeding episodes.

The limitations of this study are incomplete data of age at hemophilia diagnosis and confounding variables such as the treatment, lifestyle, and recall bias for bleeding. People with severe hemophilia A may not remember precisely the bleeding episodes at the time of the interview. A better diary to write down the bleeding episode might be necessary.

To conclude, there is no difference in bleeding episodes in the group that has excess weight and no excess weight. However, there is a trend that the minimum bleeding episodes in the group that has excess weight are higher. Further studies are interesting to evaluate the confounding variables that may relate to the finding.

\section{References}

1. Peyvandi F, Garagiola I, Young G. The past and future of haemophilia: diagnosis, treatments, and its complications. Lancet.
2016;388(10040):187-97.

2. World Federation of Hemophilia. Report on the annual global survey 2017. Montreal, Quebec, Canada: World Federation of Hemophilia; 2018 [cited 2019 October 1]. Available from: http://www1.wfh.org/ publications/files/pdf-1714.pdf

3. Tanto C, Priantono D, Sjakti HA. Hemofilia. In: Tanto C, Liwang F, Hanifati S, Pradipta EA, editors. Kapita selekta kedokteran. $4^{\text {th }}$ ed. Jakarta: Media Aesculapius; 2014. p. 53-5.

4. Tiktinsky R, Kenet G, Dvir Z, Falk B, Heim $\mathrm{M}$, Martinowitz U, et al. Physical activity participation and bleeding characteristics in young patients with severe haemophilia. Haemophilia. 2009;15(3):695-700.

5. Soucie JM, Wang C, Siddiqi A, Kulkarni $\mathrm{R}$, Recht M, Konkle BA. The longitudinal effect of body adiposity on joint mobility in young males with Haemophilia A. Haemophilia. 2011;17(2):196-203.

6. Radzevič V, Raistenskis J, Ragelienè LR, KowaIski IM. Relationship between physical activity and functional ability in school-aged children with hemophilia. Pol Ann Med. 2013;20(1):13-8.

7. Biere-Rafi S, Haak BW, Peters $M$, Gerdes VEA, Büller HR, Kamphuisen PW. The impairment in daily life of obese haemophiliacs. Haemophilia. 2011;17(2):204-8.

8. Tuinenburg A, Biere-Rafi S, Peters $M$, Verhamme P, Peerlinck K, Kruip MJHA, et al. Obesity in haemophilia patients: effect on bleeding frequency, clotting factor concentrate usage, and haemostatic and fibrinolytic parameters. Haemophilia. 2013;19(5):744-52.

9. Wong TE, Majumdar S, Adams E, Bergman S, Damiano M Lou, Deutsche J, et al. Overweight and obesity in hemophilia: a systematic review of the literature. Am J Prev Med. 2011;41(6 Suppl 4):S369-75.

10. Pocoski J, Ma A, Kessler CM, Boklage $S$, Humphries TJ. Cardiovascular comorbidities are increased in U.S. patients with haemophilia A: a retrospective database analysis. Haemophilia. 2014;20(4):472-8.

11. Septarini AD, Windiastuti E. Terapi profilaksis versus on-demand pada pasien hemofilia berat dengan hemartrosis. Sari Pediatri. 2016;11(5):311-6.

12. Zimmerman B, Valentino LA. Hemophilia: in review. Pediatr Rev. 2013;34(7):289-95.

13. Saxena K. Barriers and perceived limitations to early treatment of 
hemophilia. J Blood Med. 2013;4:49-56.

14. White W, Christie B, Orto C. Common bleeding episodes. New York: National Hemophilia Foundation; 2013 [cited
2019 October 1] Available from: https:// www.hemophilia.org/sites/default/files/ document/files/nurses-guide-chapter-4common-bleeding-episodes.pdf 